PROCEEDINGS OF THE

AMERICAN MATHEMATICAL SOCIETY

Volume 136, Number 8, August 2008, Pages 2829-2833

S 0002-9939(08)09318-0

Article electronically published on April 2, 2008

\title{
AN ELEMENTARY APPROACH TO SEVERAL RESULTS ON THE HARDY-LITTLEWOOD MAXIMAL OPERATOR
}

\author{
ANDREI K. LERNER
}

(Communicated by Michael T. Lacey)

\begin{abstract}
We give new elementary proofs of theorems due to B. Muckenhoupt, B. Jawerth, and S. Buckley. By means of our approach we answer a question raised by J. Orobitg and C. Pérez.
\end{abstract}

\section{INTRODUCTION}

Let $w$ be a weight, i.e., $w \geq 0$ and $w \in L_{\mathrm{loc}}^{1}\left(\mathbb{R}^{n}\right)$. Given a measurable set $E$, let $w(E)=\int_{E} w(x) d x$. The Hardy-Littlewood maximal operator with respect to $w$ is defined by

$$
M_{w} f(x)=\sup _{Q \ni x} \frac{1}{w(Q)} \int_{Q}|f(y)| w(y) d y,
$$

where the supremum is taken over all cubes $Q \subset \mathbb{R}^{n}$ containing $x$. If the supremum is taken only over cubes $Q$ centered at $x$, denote the corresponding operator by $M_{w}^{c}$. We drop the subscript $w$ if $w \equiv 1$. Given a weight $w$ and $p>1$, set $\sigma=w^{-1 /(p-1)}$. We say that $w$ satisfies the $A_{p}$ condition if

$$
\|w\|_{A_{p}} \equiv \sup _{Q} \frac{w(Q) \sigma(Q)^{p-1}}{|Q|^{p}}<\infty .
$$

In 8, B. Muckenhoupt proved the following fundamental result.

Theorem A. The Hardy-Littlewood maximal operator $M$ is bounded on $L_{w}^{p}, 1<$ $p<\infty$, if and only if $w \in A_{p}$.

The original proof of this theorem was based on the deep property of $A_{p}$ weights saying that $A_{p}$ implies $A_{p-\varepsilon}$ for some $\varepsilon>0$. Then R. Coifman and C. Fefferman 3 , gave a simplified proof but still depended on this property. Later, E. Sawyer [10] solved the two weight problem for M. In [6], R. Hunt, D. Kurtz and C. Neugebauer established that in the case of equal weights Sawyer's condition is equivalent to the $A_{p}$ condition, providing a new proof of Muckenhoupt's theorem that completely avoids the implication $A_{p} \Rightarrow A_{p-\varepsilon}$. After that, a very simple proof was given by M. Christ and R. Fefferman [2]. However, the proof in 2] is based essentially on

Received by the editors January 29, 2007.

2000 Mathematics Subject Classification. Primary 42B25.

Key words and phrases. Maximal operators, weights.

This work was supported by research grant SB2004-0169 from the Ministerio de Educación y Ciencia (Spain). 
the Calderón-Zygmund decomposition which makes it applicable only to classical maximal operators.

In 7], B. Jawerth obtained an even more elementary proof with the advantage that it can be applied to maximal operators with respect to a general basis $\mathcal{B}$. By a basis we mean a collection of open sets in $\mathbb{R}^{n}$. Assume that the supremum is taken in (11) and (2) over $B \in \mathcal{B}$ instead of cubes. Denote the corresponding objects by $M_{w, \mathcal{B}} f$ and $A_{p, \mathcal{B}}$.

Theorem B. Let $1<p<\infty$. Then $M_{\mathcal{B}}$ is bounded on $L_{w}^{p}$ and on $L_{\sigma}^{p^{\prime}}$ if and only if $w \in A_{p, \mathcal{B}}, M_{\sigma, \mathcal{B}}$ is bounded on $L_{\sigma}^{p}$, and $M_{w, \mathcal{B}}$ is bounded on $L_{w}^{p^{\prime}}$, where $1 / p+1 / p^{\prime}=1$.

Observe that $w \in A_{p, \mathcal{B}}$ if and only if $\sigma \in A_{p^{\prime}, \mathcal{B}}$. Next, in the case where $\mathcal{B}$ consists of cubes, the $A_{p}$ condition implies the doubling condition (there exists $c>0$ such that $w(2 Q) \leq c w(Q)$ for any cube $Q)$, and hence the boundedness of $M_{\sigma}$ on $L_{\sigma}^{p}$, and $M_{w}$ on $L_{w}^{p^{\prime}}$ follows from $A_{p}$ in the standard way by the usual covering argument. Thus, Theorem B contains Theorem A as a particular case.

In [1], S. Buckley found the sharp dependence of $\|M\|_{L_{w}^{p}}$ on $\|w\|_{A_{p}}$ in Muckenhoupt's theorem.

Theorem C. Let $1<p<\infty$. Then $\|M\|_{L_{w}^{p}} \leq c_{p, n}\|w\|_{A_{p}}^{1 /(p-1)}$, and the exponent $1 /(p-1)$ is best possible.

The original proof of this result is based on the property $A_{p} \Rightarrow A_{p-\varepsilon}$ and on interpolation. It was mentioned in [1] that some proofs of the boundedness of $M$, for instance, Jawerth's proof [7, do not yield the sharp exponent $1 /(p-1)$.

In this short note we give an extremely simple argument leading to the proofs of both Theorems B and C. This yields a new proof of Theorem A as well. Also we consider similar questions for $A_{p}$ weights with respect to non-doubling measures.

Let $\mu$ be a non-negative Radon measure in $\mathbb{R}^{n}$ with the property that $\mu(\partial Q)=0$ for any cube $Q$. Replacing in (11) $w$ by $\mu$ (and $w(y) d y$ by $d \mu(y)$ ), we get the maximal operator $M_{\mu}$. As usual, by $M_{\mu}^{c}$ we denote the centered maximal operator. Given a measurable set $E$ and a weight $w$, let $w_{\mu}(E)=\int_{E} w(x) d \mu(x)$. We say that $w$ satisfies the $A_{p}^{(r)}(\mu)(0<r \leq 1, p>1)$ condition if

$$
\|w\|_{A_{p}^{(r)}(\mu)} \equiv \sup _{Q} A_{p}^{(r)}(Q)=\frac{w_{\mu}(r Q)}{\mu(r Q)}\left(\frac{\sigma_{\mu}(Q)}{\mu(Q)}\right)^{p-1}<\infty .
$$

If $r=1$ we simply write $A_{p}(\mu)$. Denote by $L_{w}^{p}(\mu)$ the space of all measurable $f$ such that

$$
\|f\|_{L_{w}^{p}(\mu)} \equiv\left(\int_{\mathbb{R}^{n}}|f(x)|^{p} w(x) d \mu(x)\right)^{1 / p}<\infty .
$$

In [9], J. Orobitg and C. Pérez proved the following variant of Muckenhoupt's theorem.

Theorem D. If $w \in A_{p}(\mu), p>1$, then $M_{\mu}^{c}$ is bounded on $L_{w}^{p}(\mu)$.

Using the same idea as was used in proving Theorems B and C, we get that $M_{\mu}^{c}$ is also bounded on $L_{w}^{p}(\mu)$ for $A_{p}^{(r)}(\mu)$ weights, $r<1$.

Theorem $\mathbf{D}^{\prime}$. Let $0<r<1$. If $w \in A_{p}^{(r)}(\mu), p>1$, then $M_{\mu}^{c}$ is bounded on $L_{w}^{p}(\mu)$. 
It was asked in [9] whether the $A_{p}(\mu)$ condition is necessary for the $L_{w}^{p}(\mu)$ boundedness of $M_{\mu}^{c}$ as in the classical case. We show (see Example 1 in Section 2 below) that $A_{p}^{(r)}(\mu) \not \subset A_{p}(\mu), r<1$. Therefore, Theorem $\mathrm{D}^{\prime}$ provides a negative answer to this question.

In the next section we prove Theorems $\mathrm{B}, \mathrm{C}$ and $\mathrm{D}^{\prime}$. To be more precise, we prove only the difficult parts of Theorems B and C, that is, the sufficiency part in Theorem B and the estimate for $\|M\|_{L_{w}^{p}}$ in Theorem C.

\section{Proofs}

Proof of Theorem B. By the symmetry between $w$ and $\sigma$, it suffices to prove that $M_{\mathcal{B}}$ is bounded on $L_{w}^{p}$. Setting $A_{p}(B)=w(B) \sigma(B)^{p-1} /|B|^{p}$, we have

$$
\begin{aligned}
\frac{1}{|B|} \int_{B}|f| & =A_{p}(B)^{\frac{1}{p-1}}\left\{\frac{|B|}{w(B)}\left(\frac{1}{\sigma(B)} \int_{B}|f|\right)^{p-1}\right\}^{\frac{1}{p-1}} \\
& \leq\|w\|_{A_{p, \mathcal{B}}^{\frac{1}{p-1}}}\left\{\frac{1}{w(B)} \int_{B} M_{\sigma, \mathcal{B}}\left(f \sigma^{-1}\right)^{p-1} d x\right\}^{\frac{1}{p-1}},
\end{aligned}
$$

and hence,

$$
M_{\mathcal{B}} f(x) \leq\|w\|_{A_{p, \mathcal{B}}}^{\frac{1}{p-1}} M_{w, \mathcal{B}}\left(M_{\sigma, \mathcal{B}}\left(f \sigma^{-1}\right)^{p-1} w^{-1}\right)(x)^{\frac{1}{p-1}} .
$$

Therefore,

$$
\begin{aligned}
\left\|M_{\mathcal{B}} f\right\|_{L_{w}^{p}} & \leq\|w\|_{A_{p, \mathcal{B}}}^{\frac{1}{p-1}}\left\|M_{w, \mathcal{B}}\left(M_{\sigma, \mathcal{B}}\left(f \sigma^{-1}\right)^{p-1} w^{-1}\right)\right\|_{L_{w}^{p^{\prime}}}^{\frac{1}{p-1}} \\
& \leq\|w\|_{A_{p, \mathcal{B}}}^{\frac{1}{p-1}}\left\|M_{w, \mathcal{B}}\right\|_{L_{w}^{p^{\prime}}}^{\frac{1}{p-1}}\left\|M_{\sigma, \mathcal{B}}\left(f \sigma^{-1}\right)\right\|_{L_{\sigma}^{p}} \\
& \leq\|w\|_{A_{p, \mathcal{B}}}^{\frac{1}{p-1}}\left\|M_{w, \mathcal{B}}\right\|_{L_{w}^{p^{\prime}}}^{\frac{1}{p-1}}\left\|M_{\sigma, \mathcal{B}}\right\|_{L_{\sigma}^{p}}\|f\|_{L_{w}^{p}} .
\end{aligned}
$$

This completes the proof.

Observe that although the original proof of this theorem 7] (the same proof can be found in [4. p. 423]) was also simple, it involved several additional ingredients such as a selection process for sets $B_{j}^{k}$ corresponding to the level set $\left\{2^{k}<M_{\mathcal{B}} f \leq\right.$ $\left.2^{k+1}\right\}$ with the subsequent introduction of an auxiliary discrete measure.

Proof of Theorem C. Suppose that $\mathcal{B}$ is formed by cubes. Note first that in the one-dimensional case $\left\|M_{w}\right\|_{L_{w}^{p^{\prime}}}$ and $\left\|M_{\sigma}\right\|_{L_{\sigma}^{p}}$ are bounded uniformly in $w$ (see [11]). Hence, in this case (3) immediately yields the desired estimate for $\|M\|_{L_{w}^{p}}$.

In the multi-dimensional case we have only to slightly modify the above argument. Setting $A_{p}(Q)=w(Q) \sigma(3 Q)^{p-1} /|Q|^{p}$, we have

$$
\begin{aligned}
\frac{1}{|Q|} \int_{Q}|f| & =A_{p}(Q)^{\frac{1}{p-1}}\left\{\frac{|Q|}{w(Q)}\left(\frac{1}{\sigma(3 Q)} \int_{Q}|f|\right)^{p-1}\right\}^{\frac{1}{p-1}} \\
& \leq 3^{n p}\|w\|_{A_{p}}^{\frac{1}{p-1}}\left\{\frac{1}{w(Q)} \int_{Q} M_{\sigma}^{c}\left(f \sigma^{-1}\right)^{p-1} d x\right\}^{\frac{1}{p-1}} .
\end{aligned}
$$

From this and from the fact that $M f(x) \leq 2^{n} M^{c} f(x)$ we get

$$
M f(x) \leq 2^{n} 3^{n p}\|w\|_{A_{p}}^{\frac{1}{p-1}} M_{w}^{c}\left(M_{\sigma}^{c}\left(f \sigma^{-1}\right)^{p-1} w^{-1}\right)(x)^{\frac{1}{p-1}} .
$$


Using this inequality and the well-known fact (based on the Besicovitch covering theorem) that $\left\|M_{w}^{c}\right\|_{L_{w}^{p^{\prime}}}$ and $\left\|M_{\sigma}^{c}\right\|_{L_{\sigma}^{p}}$ are bounded uniformly in $w$, exactly as above, we get Buckley's theorem.

Proof of Theorem $\mathrm{D}^{\prime}$. Define the maximal operator $M_{\mu}^{(r)}$ by

$$
M_{\mu}^{(r)} f(x)=\sup _{r Q \ni x} \frac{1}{\mu(Q)} \int_{Q}|f(y)| d \mu(y)
$$

where the supremum is taken over all cubes $Q$ such that $x \in r Q$. By the Besicovitch covering theorem (see [5. pp. 6-7]) we have that the operator $M_{\mu}^{(r)}$ is bounded on $L_{\mu}^{p}$ for all $p>1$, as the usual centered maximal operator (here we essentially use that $r<1)$. We have

$$
\begin{aligned}
\frac{1}{\mu(Q)} \int_{Q}|f| d \mu & =A_{p}^{(r)}(Q)^{\frac{1}{p-1}}\left\{\frac{\mu(r Q)}{w_{\mu}(r Q)}\left(\frac{1}{\sigma_{\mu}(Q)} \int_{Q}|f| d \mu\right)^{p-1}\right\}^{\frac{1}{p-1}} \\
& \leq\|w\|_{A_{p}^{(r)}(\mu)}^{\frac{1}{p-1}}\left\{\frac{1}{w_{\mu}(r Q)} \int_{r Q} M_{\sigma(d \mu)}^{(r)}\left(f \sigma^{-1}\right)^{p-1} d \mu\right\}^{\frac{1}{p-1}},
\end{aligned}
$$

and hence,

$$
M_{\mu}^{c} f(x) \leq\|w\|_{A_{p}^{(r)}(\mu)}^{\frac{1}{p-1}} M_{w(d \mu)}^{c}\left(M_{\sigma(d \mu)}^{(r)}\left(f \sigma^{-1}\right)^{p-1} w^{-1}\right)(x)^{\frac{1}{p-1}} .
$$

From this, using the fact that $M_{w(d \mu)}^{c}$ is bounded on $L_{w}^{p^{\prime}}(\mu)$ and $M_{\sigma(d \mu)}^{(r)}$ is bounded on $L_{\sigma}^{p}(\mu)$, we get the $L_{w}^{p}(\mu)$-boundedness of $M_{\mu}^{c}$.

Remark. It is well-known (see [12] and [13]) that for $n \geq 2$ the maximal operator $M_{\mu}$ does not bounded on $L_{\mu}^{p}$, in general. Therefore, the above proof does not work when $n \geq 2$ and $r=1$. Note that the proof of Theorem D [9] was based on the property $A_{p}(\mu) \Rightarrow A_{p-\varepsilon}(\mu)$. We do not know how to avoid this property in the multi-dimensional case. On the other hand, in the case $n=1$ and $r=1$ a full analogue of inequality (4) for non-centered maximal functions holds which shows that the $A_{p}(\mu)$ condition is sufficient (and trivially necessary) for the boundedness of $M_{\mu}$ on $L_{w}^{p}(\mu)$.

In order to show that $A_{p}^{(r)}(\mu) \not \subset A_{p}(\mu), r<1$, we give the following example.

Example 1. Let $n=1$. Set $d \mu=e^{|x|} d x$ and $w(x)=e^{(p-1)|x|}$. Let us show that $w \in A_{p}^{(r)}(\mu)$ for any $r<1$ but $w \notin A_{p}(\mu)$. Indeed, it suffices to estimate $A_{p}^{(r)}(I)$ for any $I \subset(0, \infty)$. Let $I=(a, a+h)$. A straightforward calculation shows that

$$
A_{p}^{(r)}(I)=\frac{h^{p-1}}{p} \frac{e^{(p-1)(1-r) h / 2}\left(e^{p r h}-1\right)}{\left(e^{r h}-1\right)\left(e^{h}-1\right)^{p-1}} .
$$

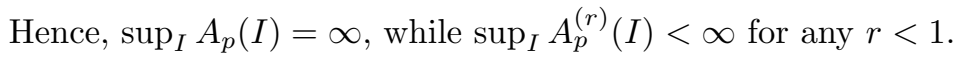

\section{ACKNOWLedGement}

This work was done during my stay at the University of Seville. I would like to thank Carlos Pérez for his hospitality. 


\section{REFERENCES}

[1] S.M. Buckley, Estimates for operator norms on weighted spaces and reverse Jensen inequalities, Trans. Amer. Math. Soc. 340 (1993), no. 1, 253-272. MR1124164 (94a:42011)

[2] M. Christ and R. Fefferman, A note on weighted norm inequalities for the Hardy-Littlewood maximal operator, Proc. Amer. Math. Soc. 87 (1983), 447-448. MR684636 (84g:42017)

[3] R.R. Coifman and C. Fefferman, Weighted norm inequalities for maximal functions and singular integrals, Studia Math. 51 (1974), 241-250. MR0358205 (50:10670)

[4] J. García-Cuerva and J.L. Rubio de Francia, Weighted norm inequalities and related topics, North-Holland Math. Stud. 116, North-Holland, Amsterdam, 1985. MR807149 (87d:42023)

[5] M. de Guzman, Differentiation of integrals in $\mathbb{R}^{n}$, Lecture Notes in Math. 481, SpringerVerlag, 1975. MR0457661 (56:15866)

[6] R. Hunt, D. Kurtz and C. Neugebauer, A note on the equivalence of $A_{p}$ and Sawyer's condition for equal weights, Proc. Conf. on Harmonic Analysis in honour of A. Zygmund, Wadsworth Math. Ser., vol. 1, pp. 156-158. MR730066 (85f:42032)

[7] B. Jawerth, Weighted inequalities for maximal operators: Linearization, localization and factorization, Amer. J. Math. 108 (1986), 361-414. MR833361 (87f:42048)

[8] B. Muckenhoupt, Weighted norm inequalities for the Hardy maximal function, Trans. Amer. Math. Soc. 165 (1972), 207-226. MR0293384 (45:2461)

[9] J. Orobitg and C. Pérez, $A_{p}$ weights for nondoubling measures in $R^{n}$ and applications, Trans. Amer. Math. Soc. 354 (2002), no. 5, 2013-2033. MR.1881028 (2002k:42044)

[10] E.T. Sawyer, A characterization of a two-weight norm inequality for maximal operators, Studia Math. 75 (1982), 1-11. MR676801 (84i:42032)

[11] P. Sjögren, A remark on the maximal function for measures in $\mathbb{R}^{n}$, Amer. J. Math. 105 (1983), 1231-1233. MR714775 (86a:28003)

[12] P. Sjögren and F. Soria, Sharp estimates for the non-centered maximal operator associated to Gaussian and other radial measures, Adv. Math. 181 (2004), no. 2, 251-275. MR2026859 (2004k:42035)

[13] A. Vargas, On the maximal function for rotation invariant measures in $\mathbb{R}^{n}$, Studia Math. 110 (1994), no. 1, 9-17. MR.1279371 (95e:42019)

Department of Mathematics, Bar-Ilan University, 52900 Ramat Gan, Israel

Current address: Departamento de Análisis Matemático, Facultad de Matemáticas, Universidad de Sevilla, 41080 Sevilla, Spain

E-mail address: aklerner@netvision.net.il 